\title{
Clinical utility of diagnostic markers for malignant pleural mesothelioma
}

\author{
B.D. Grigoriu1,2, C. Grigoriu2,3, B. Chahine4,6, \\ T. Gey5, A. Scherpereel 1,6
}

ABSTRACT: Clinical utility of diagnostic markers for malignant pleural mesothelioma. B.D. Grigoriu, C. Grigoriu, B. Chahine, T. Gey, A. Scherpereel.

Malignant mesothelioma has a very dismal prognosis with very few patients surviving one year after diagnosis. Early multimodal treatment, however, is expected to improve the outcome. Today, there is a strong need to have disease markers which could be used for screening, diagnosing, and/or monitoring tumour response to treatment. Old markers such as hyaluronic acid, various cytokeratin fragments (CYFRA 21.1, TPA) and other cancer antigens (CA 15.3, CA 125 or CA 19.9 or CEA) are not sensitive or specific enough and cannot be used in practice. More recently new molecules, such as soluble mesothelin and osteopontin, have been proposed for diagnostic purposes. Soluble mesothelin has a good specificity but has a sub- optimal sensitivity being negative in all sarcomatoid and in up to one half of epithelioid mesothelioma. On the contrary osteopontin has an inadequate specificity. Combining different markers together does not lead to an improvement in diagnostic accuracy. Neither marker can be used for screening purposes, the main limitation being the very low incidence of the disease in the at-risk, asbestos exposed population. Mesothelin is also a promising marker for monitoring response to treatment but published data is still insufficient to make recommendations. There is still a strong need for research is this area both in order to discover new markers as well as to correct the positioning of each existing molecule (alone or in combination) is the evaluation of the patients with a mesothelioma.

Monaldi Arch Chest Dis 2009; 71: 1, 31-38.

Keywords: Mesothelioma, Diagnosis, Markers, Pleural diseases, Peripheral blood.

I INSERM, U774, Institut Pasteur de Lille, France.

2 University of Medicine, Iasi, Romania.

3 Department of Pathology, Forensic Institute, Iasi, Romania.

4 Pulmonary Department, Hospital of Douai, France.

5 Pulmonary Department, Teissier Clinic, Valenciennes, France.

6 Pulmonary and Thoracic Oncology Department, Hospital of University (CHRU) of Lille II, France.

Correspondence: Dr Bogdan Dragos Grigoriu, Clinic of Pulmonary Diseases, University of Medicine and Pharmacy, Str Dr. ICihacnr 30,700115, Iasi,_Romania; e-mail:b_grigoriu@hotmail.com

\section{Introduction}

Malignant pleural mesothelioma (MPM) is a disease with an increasing incidence and a very dismal prognosis, with a median survival after diagnosis of less than one year [1]. It is estimated that the disease accounts for 15,000 to 20,000 deaths per year worldwide. In almost all male cases (versus less than 50\% female cases), MPM occurs 30 to 40 years after an occupational asbestos exposure. This occupational hazard was recognised a long time ago [2] and was followed by a interdiction of asbestos use in Iceland, Norway, Denmark and Sweden in the 80's followed by Austria, Netherlands, Finland, Italy and Germany in the early 90's by France in 1997 and by the EU ban in 1999. Despite a rapidly diminishing worldwide consumption and efforts to ban asbestos worldwide, this material is still used in many emerging countries.

Today there is a strong impulse from patients to develop new diagnostic and therapeutic modalities as well as to offer financial compensation to affected individuals. Currently, chemotherapy can offer only a small improvement in survival [3] but surgery in very few selected cases in the early phase of the disease may offer a significant chance of prolonged survival [4]. However the main problem is that most cases are diagnosed in advanced stages of the disease when very few therapeutic options are available. Diagnosing mesothelioma is not an easy task mainly because of a non specific clinical picture. No clinical elements can clearly differentiate MPM from benign pleural lesions in asbestos-exposed subjects or from pleural metastases of various carcinomas. A history of previous exposure to asbestos may orientate the diagnosis but it is documented in only 30 to $90 \%$ of patients depending on the methodology used, and is almost twice less frequently found in women than in men [5]. In accordance with the recent mesothelioma international panel guidelines, cytology of the pleural fluid is not sensitive nor specific enough for MPM diagnosis [6], and in some cases a significant pleural effusion is lacking. Multiple pleural biopsies, generally obtained by thoracoscopy, 
are required for an histological and immunohistochemical diagnosis of MPM, including tumour sub-typing [7].

Despite the strong relationship between asbestos exposure and mesothelioma, the incidence of the disease is low even among the highly exposed individuals. Therefore screening is expected to be very difficult, imposing tools with a very high specificity in order to avoid a large number of false positive results. Moreover, one can argue that MPM screening may not be currently justified because there is no available validated curative treatment for this tumour yet. However since surgery in early phases [4] and early chemotherapy [8] delays progression and may prolong survival there is a strong incentive to diagnose the disease earlier. Last but not least, there is a strong need to evaluate prognosis and response to treatment because actual imagistic techniques as CT or PET-CT are cumbersome and their practical utility is still under investigation [9-12].

Therefore in recent years markers have been searched in order to help diagnosis, prognosis and response to treatment assessment or even screening of the disease. There are several potentially good candidates but as yet none of them have been proven to be both sensitive and specific enough to be used in routine clinical practice.

\section{"Old" candidate markers}

One of the first markers to be studied was the Carcinoembryonic antigen (CEA) which has a moderate sensitivity (around 50\%) for diagnosing a malignant pleural effusion. In MPM, both blood and pleural CEA are always negative, and an elevation of CEA excludes a mesothelioma in the case of a confirmed malignant pleural effusion diagnosed on pleural fluid cytology [13]. Serum levels of CEA in MPM are similar to those of control "undiseased" subjects. In various investigations pleural values of CEA have only slightly increased but this is only generally not statistically significant in relation to the values from control cases. Thus CEA can be used only as a negative marker i.e. to exclude MPM.

Hyaluronic acid (HA) has been proposed as a putative diagnostic marker because its level is increased in approximately $60 \%$ of pleural effusions from patients with mesothelioma [14, 15]. However, elevated serum HA levels have been described only in advanced stage mesothelioma [16] and a significant percentage of MPM may not secrete HA $[17,18]$. The specificity of this marker is quite good and a high pleural level of HA (higher than $100 \mathrm{mg} / \mathrm{L}$ ) has been considered to be diagnostic for MPM. Despite a quite high area under the Receives Operating Characteristic (ROC) curve (around 0.75), the sensitivity of the test (in pleural fluid) is only about $40 \%$ to $70 \%$ at most [14, 1922]. The hyaluronic acid values in plasma are grossly 1000 times lower than those in pleural fluid, the sensitivity of blood HA is lower than in pleural fluid and therefore its practical utility is limited [16]. Hyaluronic acid was also proposed as an independent prognostic marker but to date the data supporting such use is limited [14]. More recently, pleural HA was proposed in addition to cytological examination as a minimal invasive technique for MPM diagnosis enabling an increase in sensitivity from 50 to $75 \%$ without compromising specificity [23]. Globally, despite the fact that a new ELISA-like assay has been commercialised for HA thus offering an easier method of dosage than the older HPLC techniques, the usefulness in practice is limited and is gradually supplanted by Soluble mesothelin assay which seems a more sensitive diagnostic marker for MPM (personal unpublished results).

Various cytokeratins fragments (CYFRA 21.1, tissue polypeptide antigen - TPA) and other cancer antigens (CA 15.3, CA 125 or CA 19.9) have been studied as an aid in the diagnosis of mesothelioma [19, 24-29]. Cyfra 21.1 has been shown to be useful in some studies to diagnose malignant pleural effusions [24] but no definitive cut-off value has been established. However the capacity of Cyfra 21.1 to distinguish between mesothelioma and other pleural malignancies is limited. The same situation applies to TPA which could eventually differentiate between benign and malign pleural effusions but is unable to differentiate MPM from other pleural malignancies. Globally the available data in literature for all these markers is not convincing: the case series are either limited in numbers or do not contain enough non neoplastic/non mesothelioma controls or the specificity of the studied marker is poor. Therefore these markers cannot be used alone in the diagnosis of mesothelioma but could eventually be associated with other more specific markers in an attempt to improve their sensitivity/specificity. However until now there is no convincing data to demonstrate that such a combination would be useful in practice. CA 125 was proposed as an alternative diagnostic marker for MPM but its sensitivity (below 30\%) is insufficient. Moreover the combination of CA 125 with other more specific/sensitive markers (as for example soluble mesothelin) does not significantly improve the diagnostic capabilities over the markers used alone [30].

Finally, Cyfra 21.1 and TPA were also proposed as prognostic markers but similar to HA, published data is very limited, precluding any definitive conclusion about their real utility for this purpose [31].

\section{"New" soluble markers for malignant pleural mesothelioma}

More recently the interest in the research for new diagnostic marker for MPM has been captured by two new candidates: mesothelin and osteopontin.

\section{Mesothelin}

Mesothelin is a cell-surface glycophosphatidylinositol (GPI) anchored protein which is expressed at a low level by all mesothelial cells $[32,33]$. It is se- 
creted as a $69 \mathrm{kD}$ precursor which is afterwards enzymatically cleaved by furine into a $40 \mathrm{kD}$ membrane linked protein (referred as mesothelin) and a $31 \mathrm{kD}$ soluble fragment named Megakaryocyte Potentiating Factor (or MPF). To date, the physiologic role of these two molecules is not completely understood. Mesothelin-KO mice have been generated and are viable without obvious abnormalities [34]. An analogue of mesothelin has also been described in rats and termed ERC (expressed in renal carcinoma) [35]. Mesothelin is believed to be involved in the intercellular adhesion process and interacts with the CA 125 antigen (which is part of the MUC16 glycoprotein), an interaction that may be involved in the metastatic spread of ovarian tumour cells [36], while MPF stimulates the proliferation of megakaryocytes [37]. Membrane-bound mesothelin is also found expressed by ovarian, pancreatic, colon breast and non small cell lung cancer tumour cells [38, 39] as well as by the epithelial subtype of malignant mesothelioma [33]. It is variably expressed by mixed type MPM and is not expressed by sarcomatoid MPM subtype. Soluble mesothelin can be found in the serum of some patients having a mesothelinexpressing tumour. The mechanism of release of mesothelin from the tumour-cell membrane is still not completely understood and hence this serum protein has been termed Soluble Mesothelin Related Protein or SMRP. Presence of mesothelin in pleural fluid and blood could result either from the synthesis of a mesothelin variant following a mutation or from a proteolytic cleavage from the membrane. Supporting the first hypothesis, two variants of mesothelin with different N-terminal structures have been described but they represent less than $5 \%$ of mesothelin transcripts, and some authors hypothesised that they could be the result of a sequencing error or the cloning of an incompletely processed mRNA [40]. On the other side, it has been demonstrated that mesothelin is cleaved from the membrane but the mechanism (and the precise enzymes involved) is unknown [41-43]. There is no common agreement on how this molecule should be named: currently SMRP, soluble mesothelin or $\mathrm{N}-\mathrm{ERC} /$ mesothelin are used in the literature and referred to the same molecule. For simplicity we used the term of soluble mesothelin throughout this article.

Antibodies against mesothelin were generated $[38,44,45]$ and to date at least two ELISA assays are available, having the brand name of MESOMARK $^{\mathrm{TM}}$ by Fujirebio diagnostics (antibodies developed by Scholler et al [38]) and one by Immuno-Biological Laboratories under the name of C-ERC/Mesothelin with antibodies developed by Shiomi et al [46]. The data available for the second assay is limited and it is difficult to say if this assay is better than the Mesomark ${ }^{\mathrm{TM}}$ kit which has been used by the majority of investigators and showed a very good reproducibility and precision with little constraints concerning sample storage or interference with other substances [47-49]. A head to head comparison of the two kits would be useful but is presently unavailable.

Two main diagnostic applications have been foreseen for mesothelin. The first one concerns its use as an immunohistochemical marker. Histological diagnosis of MPM is sometimes very difficult and immunohistochemistry using multiple (at least two positive and two negative) markers is necessary in order to provide a reliable diagnosis [50]. Despite the fact that mesothelin is intensely expressed by epithelioid mesotheliomas, its usefulness as a tumour tissue marker are very limited in practice since it is also expressed by normal mesothelial cells and by 30 to $40 \%$ of non small cell lung carcinomas [51]. Thus mesothelin as a tissue marker is unable to differentiate between MPM and benign mesothelial proliferations or pleural metastases of lung cancers. Moreover 20 to $30 \%$ of epithelioid MPM are not expressing mesothelin [52] as well as all sarcomatoid MPM's [51]. Therefore its usefulness is restricted to rare situations when a panel of other markers do not give a concluding result or in some very peculiar (or "niche") applications [53-55]. Mesothelin is also expressed in pancreatic carcinoma $[56,57]$ and its detection can be used for diagnosis in both histologic and cytological examinations [58-61]. Ovarian carcinomas also express mesothelin in their great majority [57, 62, 63] and mesothelin can be used for diagnostic purposes but negative cases have been described [62, 64]. Serum mesothelin values seem to be higher in ovarian cancers than in benign ovarian tumours and may be correlated with prognosis [65] but there is no agreement concerning its prognostic utility [66].

Soluble mesothelin has been also proposed for the diagnosis of MPM and the evaluation of the patients prognosis. The first results were published by Robinson et al $[67,68]$ and suggested a very high diagnostic specificity (>95\%) of serum mesothelin for the diagnosis of epithelioid subtype of MPM. The test was negative is all sarcomatoid mesothelioma as well as in various exsudative or transudative pleural effusions or other pulmonary or pleural neoplasia]. Interestingly high serum mesothelin levels have been found in seven patients exposed to asbestos of which three were subsequently diagnosed with MPM, thus suggesting its use for early MPM diagnosis. Moreover serum mesothelin levels were correlated with tumour volume and a trend toward increasing values with tumour progression was observed. Surgery resulted in a sharp decrease in serum mesothelin while chemotherapy induced no change [67]. A significantly larger series from France confirmed the diagnostic utility of mesothelin $[69,70]$ followed by similar results in series from Italy [71, 72] and Netherlands [24]. Globally analysed, the results from these different teams showed that serum mesothelin is a good diagnostic marker with areas under ROC curved around $0.8-0.85$. There is no established diagnostic cut-off value for serum mesothelin but different teams suggested values between $1 \mathrm{nmol} / \mathrm{l}$ and $1.6 \mathrm{nmol} / \mathrm{l}$ depending on their perception of the best sensitivity/specificity combination. However up to one third of mesotheliomas do not have elevated levels of mesothelin with a minimum cut-off at $1 \mathrm{nmol} / \mathrm{l}$ and up to $40-$ $50 \%$ of MPM may be considered negative if a 
specificity of at least $95 \%$ is requested. Moreover about $20 \%$ of lung adenocarcinomas have elevated levels of serum mesothelin [24, 70].

Using another pair of anti-mesothelin antibodies, Hassan et al created an ELISA assay that confirmed the good diagnostic performance of serum mesothelin in both epithelioid mesotheliomas and ovarian carcinomas [45] but this assay is not available commercially.

One way of improving the diagnostic accuracy may be to combine multiple markers in order to palliate for the lack of sensitivity or specificity of one marker. A few publications have tried to combine soluble mesothelin with other markers. To date no combination has been proved superior to soluble mesothelin alone either if combined with CA 125 [30], with hyaluronic acid or with MPF and/or osteopontin [73]. Some authors have suggested that the association of Cyfra 21.1 to soluble mesothelin may be useful [24] but the perceived utility may depend on how the data is analysed (i.e. what is the practical clinical question) and there is need of supplemental research in this area before being able to make a clear recommendation for clinical practice.

Soluble mesothelin can also be assayed in pleural effusions from malignant mesothelioma, the pleural values being grossly ten times higher than the corresponding serum values. However pleural and serum values are highly correlated, and there is no significant improvement in sensitivity or specificity using pleural mesothelin over blood assessment [70, 74].

Another potential use of soluble mesothelin is the evaluation of the disease prognosis. Indeed since mesothelin is produced by the tumour itself, serum levels should reflect the tumour burden and hence could be related to prognosis and tumour response to treatment. After the first data published by Robinson et al [67], Pass et al showed that serum mesothelin level increase with mesothelioma stage (assessed using the IMIG, International Mesothelioma Interest Group, classification) [75], decrease sharply with surgery and increases again when the tumour progresses [76]. We also showed that there is a relationship between serum mesothelin level at diagnosis and patients survival [70]. Similar results were also published by Cristaudo et al [72], but other authors did not find such relationship [74].

Contrasting with the clear decrease of serum mesothelin levels in mesothelioma patients after surgical tumour resection, there is surprisingly little data concerning the mesothelin kinetics in patients treated by chemotherapy and the value of serum mesothelin in monitoring patient's response to treatment. However, the FDA has recently approved the Mesomark, test as a humanitarian device for this purpose in the USA. We recently showed that in patients with an objective response following chemotherapy (as evaluated by the modified RECIST, Response Evaluation Criteria in Solid Tumors, criteria [9]), serum mesothelin level tends to decrease while in patients with progressive disease serum mesothelin value increases pro- gressively [77]. Moreover we showed that serum mesothelin increases in patients with an unfavourable outcome while it remains stable or decreases after therapy in patients still alive at the end of the follow-up period. Similar results were reported by Simonini et al [78]. However the analysed series were small and confirmative investigations are necessary.

A very appealing application of mesothelin assay is the early diagnosis of MPM in a phase were surgical resection (and hence potential curability) is feasible. Data from literature shows that in asbestos exposed subjects serum mesothelin is only marginally but not significantly increased over normal unexposed subjects [71] and there seems to be no increase over time [79]. Roe et al showed in a small series of 47 malignant mesothelioma for which they had available at least one serum sample, sampled between 6 months and 30 years before diagnosis that there is no significant elevation of serum mesothelin which could be useful for early mesothelioma diagnosis [80]. In this series neither CYFRA 21.1 nor CA 125 were more useful for mesothelioma early diagnosis. In a recent paper Park et al investigated a cohort of 538 asbestos exposed subjects and concluded that serum mesothelin is slightly but significantly increased in patients with pleural plaques secondary to asbestos exposure compared with healthy asbestos exposed controls [81]. However no malignant mesothelioma was detected despite the fact that 15 patients (i.e. $2.78 \%$ ) had mesothelin values exceeding 2.5 $\mathrm{nmol} / \mathrm{l}$, value which should in theory confer an almost $100 \%$ specificity for this test. These negative results are in fact a consequence of the low incidence of mesothelioma even in the heavy-exposed population (generally less than $5 \%$ [82]) which results in a high number of false positive tests despite of a theoretically very high specificity of the test. Thus it is probable that a successful mesothelioma screening programme should involve a more elaborate protocol than a simple blood test.

\section{The Megakaryocyte Potentiating Factor}

The Megakaryocyte Potentiating Factor (MPF) protein is the soluble part of the mesothelin precursor and it is released by the proteolytic cleavage by furine [37]. Since MPF release requires only the action of a physiologically active enzyme it is hoped that its sensitivity should be almost $100 \%$ for the mesothelin over-expressing tumours (i.e. almost all epithelioid and some mixed type MPM). Two ELISA systems have been developed to date. The first one by Shiomi et al (and which is commercially available from Immuno-Biological Laboratories Gunma, Japan, under the name of $\mathrm{N}$-ERC/mesothelin) was evaluated in a short series of fourteen patients with MPM which were compared with healthy controls, patients pleural metastases of lung adenocarcinomas and patients with benign pleural lesions [46]. A second test developed by Onda et al was used to evaluate 56 patients with MPM which were compared with normal controls [83]. These first investigations as well as another 
short Japanese series [84] suggested higher diagnostic capabilities than soluble mesothelin. However these encouraging results were not confirmed by a larger series of patients [73]. Thus, today mesothelin remains the most interesting diagnostic marker despite its suboptimal sensitivity.

\section{Therapeutic uses of membrane bound mesothelin}

Due to the very restricted expression of mesothelin in normal tissue and the intense over expression of the same molecule by cancer cells it will be very interesting to use mesothelin for molecular targeting of various cytotoxic products directly to the tumour cells or to use it as a target antigen for immunotherapy [85]. Since mesothelin contains several epitopes which induce cytotoxic T cell activation [86] and that after a systemic administration the distribution of anti mesothelin antibodies is made specifically to the tumour for as long as 7 days, both approaches are feasible [87]. Actually Hassan et al have constructed a fusion protein named SSP1 by combining an antimesothelin antibody and a Pseudomonas Aeruginosa immunotoxin [88]. In vitro results showed a intense cytotoxic activity against mesothelin expressing tumour cells $[89,90]$. Moreover, In vivo experiments on mouse models of cancer resulted in an antitumour activity against the primary tumour but also against metastases [88, 90, 91] as well as a synergic activity with irradiation [92] and chemotherapy [93]. Human testing generated encouraging results and Phase I trial are under way $[94,95]$. A good recent review on that matter was published recently [96].

\section{Osteopontin}

Osteopontin is a pleiotropic molecule which has been involved in non-mineral bone matrix formation, survival of malignant cells and tumour progression [97] as well as a cytokine involved in granulomatous immune response [98]. Elevated values of circulating osteopontin have been described in a wide span of neoplastic localisations from lung to breast, colon and ovarian cancers to some non tumoural pathologies as for example tuberculosis [99]. Assaying this molecule may raise some practical concerns since we have at least three kits available on the market (from Immunobiological Laboratories, Assays Design and R\&D Systems) which may not give similar results [100] but data from mesothelioma series shows similar diagnostic capabilities whichever kit is used. Due to a proteolytic cleavage by thrombin during blood clothing, serum osteopontin values are much less important than the corresponding plasma values but published data refers mainly to serum values. The broad expression of osteopontin in various situations suggests that the potential specificity of this test will be very low. However Pass et al reported that serum osteopontin is elevated in the vast majority of mesothelioma (>95\%) irrespective of their histologic subtype. In the asbestos exposed population these authors found a relationship be- tween serum osteopontin and the duration of exposure as well as with the intensity of the radiologic lesions attributable to asbestos. But there was no relationship between serum osteopontin and disease staging, the values being sharply elevated even in stage 1 mesothelioma, suggesting that this marker could be used for early diagnosis or even screening of the disease. However this series did not include patients with other pleural diseases and the real diagnostic value of this marker cannot be assessed from this study only [82, 101]. We have recently compared the diagnostic utility of osteopontin and mesothelin in a series of 172 patients suspected of MPM and 112 asbestos exposed patients [70]. We confirmed the findings of Pass et al that mesothelioma patients have higher serum osteopontin levels than the asbestos exposed population but osteopontin was unable to separate between different types of pleural malignancies (MPM vs. pleural metastases) or even between patients with pleural malignancies and patients with benign pleural lesions associated with asbestos exposure. Moreover there was no statistically significant improvement in classifying patients when using the combination of osteopontin with mesothelin versus mesothelin alone. Subsequent investigations by Creaney et al confirmed the lack of specificity of osteopontin which was unable to distinguish between different types of pleural malignancies or between different types of pleural effusions.

Intriguingly there has been no data published until now concerning the association of osteopontin with other markers for the diagnosis of mesothelioma.

Concerning the role of osteopontin as a prognostic marker there is very little data yet published. To date only one French series had shown that high serum levels of osteopontin had a negative prognostic value [70]. Recently Cappia et al showed that osteopontin expression by the tumour (quantified by immunohistochemistry) is related to survival and is an independent prognostic factor [102].

\section{Conclusions}

Today there is a strong need for one or more markers for malignant pleural mesothelioma which could be used for diagnostic, screening and/or monitoring tumour response to treatment. The new molecules investigated in the last five years, as soluble mesothelin/MPF and osteopontin, have a higher sensitivity than old markers as Hyaluronic acid. However, today, there is no "usable" marker which could be translated to clinical practice: Mesothelin is useful for diagnosis and possibly for monitoring of patients but its principal limitation is the insufficient sensitivity. Osteopontin and MPF seems not to be specific enough and will surely give more false positive than true positive results. There is still a strong need for research is this area both for discovering new markers as well as for a correct positioning of each existing molecule (alone or in combination) is the evaluation of the patients with mesotheliomas. 


\section{References}

1. Herndon JE, Green MR, Chahinian AP, et al. Factors predictive of survival among 337 patients with mesothelioma treated between 1984 and 1994 by the Cancer and Leukemia Group B. Chest 1998; 113: 723-31.

2. Wagner JC, Sleggs CA, Marchand P. Diffuse pleural mesothelioma and asbestos exposure in the North Western Cape Province. Br J Ind Med 1960; 17: 260-71.

3. Vogelzang N, Rusthoven J, Symanowski J, et al. Phase III Study of Pemetrexed in Combination With Cisplatin Versus Cisplatin Alone in Patients With Malignant Pleural Mesothelioma. J Clin Oncol 2003; 21: 26362644.

4. Sugarbaker DJ, Flores RM, Jaklitsch MT, et al. Resection margins, extrapleural nodal status, and cell type determine postoperative long-term survival in trimodality therapy of malignant pleural mesothelioma: results in 183 patients. J Thorac Cardiovasc Surg 1999; 117: 54-63.

5. Walker AM, Loughlin JE, Friedlander ER, Rothman KJ, Dreyer NA. Projections of asbestos-related disease 1980-2009; J Occup Med 1983; 25: 409-25.

6. Stahel RA, Weder W, Felip E. Malignant pleural mesothelioma: ESMO clinical recommendations for diagnosis, treatment and follow-up. Ann Oncol 2008; 19 Suppl 2: ii43-4.

7. Greillier L, Cavailles A, Fraticelli A, et al. Accuracy of pleural biopsy using thoracoscopy for the diagnosis of histologic subtype in patients with malignant pleural mesothelioma. Cancer 2007; 110: 2248-52.

8. Jassem J, Ramlau R, Santoro A, et al. Phase III trial of pemetrexed plus best supportive care compared with best supportive care in previously treated patients with advanced malignant pleural mesothelioma. J Clin Oncol 2008; 26: 1698-1704.

9. Ceresoli, G.L., A. Chiti, P.A. Zucali, et al. Assessment of tumour response in malignant pleural mesothelioma. Cancer Treat Rev 2007; 33: 533-41.

10. Francis RJ, Byrne MJ, van der Schaaf AA, et al. Early prediction of response to chemotherapy and survival in malignant pleural mesothelioma using a novel semiautomated 3-dimensional volume-based analysis of serial 18F-FDG PET scans. J Nucl Med 2007; 48: 1449-58.

11. Flores RM. The role of PET in the surgical management of malignant pleural mesothelioma. Lung Cancer 2005; 49 Suppl 1: S27-32.

12. Armato SG 3rd, Oxnard GR, MacMahon H, et al. Measurement of mesothelioma on thoracic CT scans: a comparison of manual and computer-assisted techniques. Med Phys 2004; 31: 1105-15.

13. Shi HZ, Liang QL, Jiang J, Qin XJ, Yang HB. Diagnostic value of carcinoembryonic antigen in malignant pleural effusion: a meta-analysis. Respirology 2008; 13: 518-27.

14. Thylen A, Hjerpe A, Martensson G. Hyaluronan content in pleural fluid as a prognostic factor in patients with malignant pleural mesothelioma. Cancer 2001; 92: 1224-30.

15. Hedman M, Arnberg H, Wernlund J, Riska H, Brodin O. Tissue polypeptide antigen (TPA), hyaluronan and CA 125 as serum markers in malignant mesothelioma. Anticancer Res 2003; 23: 531-6.

16. Frebourg T, Lerebours G, Delpech B, et al. Serum hyaluronate in malignant pleural mesothelioma. Cancer 1987; 59: 2104-7.

17. Boersma A, Degand P, Biserte G. Hyaluronic acid analysis and the diagnosis of pleural mesothelioma. Bull Eur Physiopathol Respir 1980; 16: 41-5.

18. Chiu B, Churg A, Tengblad A, Pearce R, McCaughey WT. Analysis of hyaluronic acid in the diagnosis of malignant mesothelioma. Cancer 1984; 54: 2195-9.
19. Fuhrman C, Duche JC, Chouaid C, et al. Use of tumour markers for differential diagnosis of mesothelioma and secondary pleural malignancies. Clin Biochem 2000; 33: 405-10.

20. Soderblom T, Pettersson T, Nyberg P, et al. High pleural fluid hyaluronan concentrations in rheumatoid arthritis. Eur Respir J 1999; 13: 519-22.

21. Atagi S, Ogawara M, Kawahara M, et al. Utility of hyaluronic acid in pleural fluid for differential diagnosis of pleural effusions: likelihood ratios for malignant mesothelioma. Jpn J Clin Oncol 1997; 27: 293-7.

22. Martensson G, Thylen A, Lindquist U, Hjerpe A. The sensitivity of hyaluronan analysis of pleural fluid from patients with malignant mesothelioma and a comparison of different methods. Cancer 1994; 73: 1406-10.

23. Welker L, Muller M, Holz O, et al. Cytological diagnosis of malignant mesothelioma - improvement by additional analysis of hyaluronic acid in pleural effusions. Virchows Arch 2007; 450: 455-61.

24. van den Heuvel MM, Korse CM, Bonfrer JM, Baas P, Non-invasive diagnosis of pleural malignancies: the role of tumour markers. Lung Cancer 2008; 59: 350-4.

25. Alatas F, Alatas O, Metintas M, et al. Diagnostic value of CEA, CA 15-3, CA 19-9, CYFRA 21-1, NSE and TSA assay in pleural effusions. Lung Cancer 2001; 31: 9-16.

26. Nisman B, Barak V, Heching N, et al. Cytokeratin markers in malignant pleural mesothelioma. Cancer Detect Prev 1998; 22: 416-21.

27. Marukawa M, Hiyama J, Shiota Y, et al. The usefulness of CYFRA 21-1 in diagnosing and monitoring malignant pleural mesothelioma. Acta Med Okayama 1998; 52: p. 119-23.

28. Bonfrer JM, Schouwink JH, Korse CM, Baas P. Cyfra 21-1 and TPA as markers in malignant mesothelioma. Anticancer Res 1997; 17: 2971-3.

29. Paganuzzi M, Onetto M, Marroni P, et al. Diagnostic value of CYFRA 21-1 tumour marker and CEA in pleural effusion due to mesothelioma. Chest 2001; 119: 1138-42.

30. Creaney J, van Bruggen I, Hof M, et al. Combined CA125 and mesothelin levels for the diagnosis of malignant mesothelioma. Chest 2007; 132: 1239-1246.

31. Schouwink H, Korse CM, Bonfrer JM, Hart AA, Baas P. Prognostic value of the serum tumour markers Cyfra 21-1 and tissue polypeptide antigen in malignant mesothelioma. Lung Cancer 1999; 25: 25-32.

32. Chang K, Pai LH, Batra JK, Pastan I, Willingham MC. Characterization of the antigen (CAK1) recognized by monoclonal antibody K1 present on ovarian cancers and normal mesothelium. Cancer Res 1992; 52: 181-6.

33. Chang K, Pastan I. Molecular cloning of mesothelin, a differentiation antigen present on mesothelium, mesotheliomas, and ovarian cancers. Proc Natl Acad Sci U S A 1996; 93: 136-40.

34. Bera TK, Pastan I. Mesothelin is not required for normal mouse development or reproduction. Mol Cell Biol 2000; 20: 2902-6.

35. Yamashita Y, Yokoyama M, Kobayashi E, Takai S, Hino O. Mapping and determination of the cDNA sequence of the Erc gene preferentially expressed in renal cell carcinoma in the Tsc2 gene mutant (Eker) rat model. Biochem Biophys Res Commun 2000; 275: 134-40.

36. Gubbels JA, Belisle J, Onda M, et al. MesothelinMUC16 binding is a high affinity, $\mathrm{N}$-glycan dependent interaction that facilitates peritoneal metastasis of ovarian tumours. Mol Cancer 2006; 5: 50.

37. Kojima T, Oh-eda M, Hattori K, et al. Molecular cloning and expression of megakaryocyte potentiating factor cDNA. J Biol Chem 1995; 270: 21984-90.

38. Scholler N, Fu N, Yang Y, et al. Soluble member(s) of the mesothelin/megakaryocyte potentiating factor fam- 
ily are detectable in sera from patients with ovarian carcinoma. Proc Natl Acad Sci U S A 1999; 96: 11531-6.

39. Chang K, Pastan I. Molecular cloning and expression of a cDNA encoding a protein detected by the K1 antibody from an ovarian carcinoma (OVCAR-3) cell line. Int $J$ Cancer 1994; 57: 90-7.

40. Muminova ZE, StrongTV, Shaw DR. Characterization of human mesothelin transcripts in ovarian and pancreatic cancer. BMC Cancer 2004; 4: 19.

41. Sapede C, Gauvrit A, Barbieux I, et al. Aberrant splicing and protease involvement in mesothelin release from epithelioid mesothelioma cells. Cancer Sci 2008; 99: 590-4.

42. Hellstrom I, Raycraft J, Kanan S, et al. Mesothelin variant 1 is released from tumour cells as a diagnostic marker. Cancer Epidemiol Biomarkers Prev 2006; 15: 1014-20.

43. Ho M, Onda M, Wang QC, et al. Mesothelin is shed from tumour cells. Cancer Epidemiol Biomarkers Prev 2006; 15: 1751 .

44. Onda M, Willingham M, Nagata S, et al. New monoclonal antibodies to mesothelin useful for immunohistochemistry, fluorescence-activated cell sorting, Western blotting, and ELISA. Clin Cancer Res 2005; 11: 5840-6.

45. Hassan R, Remaley AT, Sampson ML, et al. Detection and quantitation of serum mesothelin, a tumour marker for patients with mesothelioma and ovarian cancer. Clin Cancer Res 2006; 12: 447-53.

46. Shiomi K, Miyamoto H, Segawa T, et al. Novel ELISA system for detection of $\mathrm{N}$-ERC/mesothelin in the sera of mesothelioma patients. Cancer Sci 2006; 97: 928-32.

47. Weber DG, Taeger D, Pesch B, et al. Soluble mesothelin-related peptides (SMRP) - high stability of a potential tumour marker for mesothelioma. Cancer Biomark 2007; 3: 287-92.

48. Di Serio F, Fontana A, Loizzi M, et al. Mesothelin family proteins and diagnosis of mesothelioma: analytical evaluation of an automated immunoassay and preliminary clinical results. Clin Chem Lab Med 2007; 45: 634-8.

49. Beyer HL, Geschwindt RD, Glover CL, et al. MESOMARK: a potential test for malignant pleural mesothelioma. Clin Chem 2007; 53: 666-72.

50. Galateau-Salle F, Copin MC, Delajartre AY, et al. Which criteria for the anatomopathologic diagnosis of malignant pleural mesothelioma? Rev Mal Respir 2006; 23: 11 S37-44.

51. Ordonez NG. The diagnostic utility of immunohistochemistry in distinguishing between epithelioid mesotheliomas and squamous carcinomas of the lung: a comparative study. Mod Pathol 2006; 19: 417-28.

52. Yaziji H, Battifora H, Barry TS, et al. Evaluation of 12 antibodies for distinguishing epithelioid mesothelioma from adenocarcinoma: identification of a three-antibody immunohistochemical panel with maximal sensitivity and specificity. Mod Pathol 2006; 19: 514-23.

53. Ordonez NG. The diagnostic utility of immunohistochemistry in distinguishing between mesothelioma and renal cell carcinoma: a comparative study. Hum Pathol 2004; 35: 697-710.

54. Ordonez NG. Value of mesothelin immunostaining in the diagnosis of mesothelioma. Mod Pathol 2003; 16 : 192-7.

55. Ordonez NG. What are the current best immunohistochemical markers for the diagnosis of epithelioid mesothelioma? A review and update. Hum Pathol 2007; 38: 1-16.

56. Argani P, Iacobuzio-Donahue $\mathrm{C}$, Ryu B, et al. Mesothelin is overexpressed in the vast majority of ductal adenocarcinomas of the pancreas: identification of a new pancreatic cancer marker by serial analysis of gene expression (SAGE). Clin Cancer Res 2001; 7: 3862-8.
57. Ordonez NG. Application of mesothelin immunostaining in tumour diagnosis. Am J Surg Pathol 2003; 27 : 1418-28.

58. Jhala N, Jhala D, Vickers SM, et al. Biomarkers in Diagnosis of pancreatic carcinoma in fine-needle aspirates. Am J Clin Pathol 2006; 126: 572-9.

59. Hassan R, Laszik ZG, Lerner M, et al. Mesothelin is overexpressed in pancreaticobiliary adenocarcinomas but not in normal pancreas and chronic pancreatitis. Am $J$ Clin Pathol 2005; 124: 838-45.

60. Cao D, Maitra A, Saavedra JA, et al. Expression of novel markers of pancreatic ductal adenocarcinoma in pancreatic nonductal neoplasms: additional evidence of different genetic pathways. Mod Pathol 2005; 18: 752-61.

61. Kachali C, Eltoum I, Horton D, Chhieng DC. Use of mesothelin as a marker for mesothelial cells in cytologic specimens. Semin Diagn Pathol 2006; 23: 20-4.

62. Le Page C, Ouellet V, Madore J, et al. Gene expression profiling of primary cultures of ovarian epithelial cells identifies novel molecular classifiers of ovarian cancer. Br J Cancer 2006; 94: 436-45.

63. Drapkin R, Crum CP, Hecht JL. Expression of candidate tumour markers in ovarian carcinoma and benign ovary: evidence for a link between epithelial phenotype and neoplasia. Hum Pathol 2004; 35: 1014-21.

64. Hassan R, Kreitman RJ, Pastan I, Willingham MC. Localization of mesothelin in epithelial ovarian cancer. Appl Immunohistochem Mol Morphol 2005; 13: 243-7.

65. Huang CY, Cheng WF, Lee CN, et al. Serum mesothelin in epithelial ovarian carcinoma: a new screening marker and prognostic factor. Anticancer Res 2006; 26: 4721-8.

66. Yen MJ, Hsu CY, Mao TL, et al. Diffuse mesothelin expression correlates with prolonged patient survival in ovarian serous carcinoma. Clin Cancer Res 2006; 12: 827-31.

67. Robinson BW, Creaney J, Lake R, et al. Mesothelinfamily proteins and diagnosis of mesothelioma. Lancet 2003; 362: 1612-6.

68. Creaney J, Christansen H, Lake R, et al. Soluble Mesothelin Related Protein in Mesothelioma. J Thoracic Oncol 2006; 1: 172-174.

69. Scherpereel A, Grigoriu B, Conti M, et al. Soluble mesothelin-related peptides in the diagnosis of malignant pleural mesothelioma. Am J Respir Crit Care Med 2006; 173: 1155-60.

70. Grigoriu BD, Scherpereel A, Devos P, et al. Utility of osteopontin and serum mesothelin in malignant pleural mesothelioma diagnosis and prognosis assessment. Clin Cancer Res 2007; 13: 2928-35.

71. Amati M, Tomasetti M, Scartozzi M, et al. Profiling tumour-associated markers for early detection of malignant mesothelioma: an epidemiologic study. Cancer Epidemiol Biomarkers Prev 2008; 17: 163-70.

72. Cristaudo A, Foddis R, Vivaldi A, et al. Clinical significance of serum mesothelin in patients with mesothelioma and lung cancer. Clin Cancer Res 2007; 13: 5076-81.

73. Creaney J, Yeoman D, Demelker Y, et al. Comparison of osteopontin, megakaryocyte potentiating factor, and mesothelin proteins as markers in the serum of patients with malignant mesothelioma. J Thorac Oncol 2008; 3: 851-7.

74. Creaney J, Yeoman D, Naumoff LK, et al. Soluble mesothelin in effusions: a useful tool for the diagnosis of malignant mesothelioma. Thorax 2007; 62: 569-76.

75. Pass HI, Wali A, Tang N, et al. Soluble Mesothelin-Related Peptide Level Elevation in Mesothelioma Serum and Pleural Effusions. Ann Thor Surg 2008; 85: 265272.

76. Pass HI, Wolaniuk D, Wali A, et al. Soluble mesothelin related peptides: a potential biomarker for malignant 
pleural mesothelioma (abstract). J Clin Oncol (2005 ASCO Annual Meeting Abstracts) 2005; 23 (9532).

77. Grigoriu BD, Chahine B, Conti M, et al. Utility of serum soluble mesothelin as a follow-up marker in patients with malignant pleural mesothelioma (abstract). In European Respiratory Society Annnual Congress 2007; Stockholm.

78. Simonini S, Foddis R, Filiberti R, et al. Evaluation of a series of serum mesothelin in patients with pleural malignant mesothelioma. G Ital Med Lav Ergon 2007; 29: 339-42.

79. Foddis R, Vivaldi A, Filiberti R, et al. Serum mesothelin dosages in follow-up of previously exposed workers. G Ital Med Lav Ergon 2007; 29: 342-5.

80. Roe OD, Creaney J, Lundgren S, et al. Mesothelin-related predictive and prognostic factors in malignant mesothelioma: A nested case-control study. Lung Cancer 2008; 61: 235-243.

81. Park EK, Sandrini A, Yates DH, et al. Soluble mesothelin-related protein in an asbestos-exposed population: the dust diseases board cohort study. Am J Respir Crit Care Med 2008; 178: 832-7.

82. Cullen MR. Serum osteopontin levels - is it time to screen asbestos-exposed workers for pleural mesothelioma? N Engl J Med 2005; 353: 1617-8.

83. Onda M, Nagata S, Ho M, et al. Megakaryocyte potentiation factor cleaved from mesothelin precursor is a useful tumour marker in the serum of patients with mesothelioma. Clin Cancer Res 2006; 12: 4225-31.

84. Iwahori K, Osaki T, Serada S, et al. Megakaryocyte potentiating factor as a tumour marker of malignant pleural mesothelioma: Evaluation in comparison with mesothelin. Lung Cancer 2008; 2: 2.

85. Hassan R, BeraT, Pastan I. Mesothelin: a new target for immunotherapy. Clin Cancer Res 2004; 10: 3937-42.

86. Yokokawa J, Palena C, Arlen P, et al. Identification of novel human CTL epitopes and their agonist epitopes of mesothelin. Clin Cancer Res 2005; 11: 6342-51.

87. Hassan R, Wu C, Brechbiel MW, et al. 111Indium-labeled monoclonal antibody K1: biodistribution study in nude mice bearing a human carcinoma xenograft expressing mesothelin. Int J Cancer 1999; 80: 559-63.

88. Hassan R, Viner JL, Wang QC, et al. Anti-tumour activity of K1-LysPE38QQR, an immunotoxin targeting mesothelin, a cell-surface antigen overexpressed in ovarian cancer and malignant mesothelioma. $J$ Immunother 2000; 23: 473-9.

89. Hassan R, Lerner MR, Benbrook D, et al. Antitumour activity of SS(dsFv)PE38 and SS1(dsFv)PE38, recombinant antimesothelin immunotoxins against human gy- necologic cancers grown in organotypic culture in vitro. Clin Cancer Res 2002; 8: 3520-6.

90. Li Q, Verschraegen CF, Mendoza J, Hassan R. Cytotoxic activity of the recombinant anti-mesothelin immunotoxin, SS1(dsFv)PE38, towards tumour cell lines established from ascites of patients with peritoneal mesotheliomas. Anticancer Res 2004; 24: 1327-35.

91. Fan D, Yano S, Shinohara H. Targeted therapy against human lung cancer. Mol Cancer Ther 2002; 1: 595600 .

92. Hassan R, Williams-Gould J, Steinberg SM, et al. Tumour-directed radiation and the immunotoxin SS1P in the treatment of mesothelin-expressing tumour xenografts. Clin Cancer Res 2006; 12: 4983-8.

93. Zhang Y, Xiang L, Hassan R, et al. Synergistic antitumour activity of taxol and immunotoxin SS1P in tumourbearing mice. Clin Cancer Res 2006; 12: 4695-701.

94. Kreitman R, Squires D, O'Hagan D, et al. SS1(dsFv)PE38 anti-mesothelin immunotoxin in advanced malignancies: phase I study of continuous infusion. Proc Am Soc Clin Oncol 2002; 21: (abstr 1896).

95. Hassan R, Bullock S, Kindler H, Pastan I. Updated results of the phase I study of SS1(dsFv)PE38 for targeted therapy of mesothelin expressing cancers. Eur $J$ Cancer 2004; 2: 280a.

96. Hassan R, Ho M. Mesothelin targeted cancer immunotherapy. Eur J Cancer 2008; 44: 46-53.

97. Wai PY, Kuo PC. The role of Osteopontin in tumour metastasis. J Surg Res 2004; 121: 228-41.

98. Ashkar S, Weber GF, Panoutsakopoulou V, et al. Eta-1 (osteopontin): an early component of type-1 (cell-mediated) immunity. Science 2000; 287: 860-4.

99. Koguchi Y, Kawakami K, Uezu K, et al. High plasma osteopontin level and its relationship with interleukin12-mediated type $1 \mathrm{~T}$ helper cell response in tuberculosis. Am J Respir Crit Care Med 2003; 167: 1355-9.

100. Vordermark D, Said HM, Katzer A, et al. Plasma osteopontin levels in patients with head and neck cancer and cervix cancer are critically dependent on the choice of ELISA system. BMC Cancer 2006; 6: 207.

101. Beck A, Ivanova A, Ivanov S, et al. Evaluation of plasma osteopontin as early detection and prognostic marker in malignant pleural mesothelioma. J Clin Oncol (Meeting Abstract) 2008; 26: 11074.

102. O'Regan AW, Serlin D, Berman JS. Asbestos exposure and serum osteopontin. N Engl J Med 2006; 354.

103. Cappia S, Righi L, Mirabelli D, et al. Prognostic role of osteopontin expression in malignant pleural mesothelioma. Am J Clin Pathol 2008; 130: 58-64. 